\title{
Convalescent plasma in patients admitted to hospital with COVID-19 (RECOVERY): a randomised controlled, open-label, platform trial
}

RECOVERY Collaborative Group*

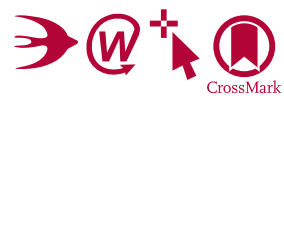

Oa

\section{Summary}

Background Many patients with COVID-19 have been treated with plasma containing anti-SARS-CoV-2 antibodies. We aimed to evaluate the safety and efficacy of convalescent plasma therapy in patients admitted to hospital with COVID-19.

Methods This randomised, controlled, open-label, platform trial (Randomised Evaluation of COVID-19 Therapy [RECOVERY]) is assessing several possible treatments in patients hospitalised with COVID-19 in the UK. The trial is underway at 177 NHS hospitals from across the UK. Eligible and consenting patients were randomly assigned (1:1) to receive either usual care alone (usual care group) or usual care plus high-titre convalescent plasma (convalescent plasma group). The primary outcome was 28-day mortality, analysed on an intention-to-treat basis. The trial is registered with ISRCTN, 50189673, and ClinicalTrials.gov, NCT04381936.

Findings Between May 28, 2020, and Jan 15, 2021, 11558 (71\%) of 16287 patients enrolled in RECOVERY were eligible to receive convalescent plasma and were assigned to either the convalescent plasma group or the usual care group. There was no significant difference in 28-day mortality between the two groups: 1399 (24\%) of 5795 patients in the convalescent plasma group and $1408(24 \%)$ of 5763 patients in the usual care group died within 28 days (rate ratio 1.00, 95\% CI 0.93-1.07; $\mathrm{p}=\mathbf{0}$.95). The 28-day mortality rate ratio was similar in all prespecified subgroups of patients including in those patients without detectable SARS-CoV-2 antibodies at randomisation. Allocation to convalescent plasma had no significant effect on the proportion of patients discharged from hospital within 28 days (3832 [66\%] patients in the convalescent plasma group vs 3822 [66\%] patients in the usual care group; rate ratio 0.99 , 95\% CI 0.94-1.03; $\mathrm{p}=\mathbf{0} \cdot 57$ ). Among those not on invasive mechanical ventilation at randomisation, there was no significant difference in the proportion of patients meeting the composite endpoint of progression to invasive mechanical ventilation or death (1568 [29\%] of 5493 patients in the convalescent plasma group vs 1568 [29\%] of 5448 patients in the usual care group; rate ratio $0 \cdot 99,95 \%$ CI $0 \cdot 93-1 \cdot 05 ; \mathrm{p}=0 \cdot 79)$.

Interpretation In patients hospitalised with COVID-19, high-titre convalescent plasma did not improve survival or other prespecified clinical outcomes.

Funding UK Research and Innovation (Medical Research Council) and National Institute of Health Research.

Copyright (C) 2021 The Author(s). Published by Elsevier Ltd. This is an Open Access article under the CC BY 4.0 license.

\section{Introduction}

A substantial proportion of individuals with SARS-CoV-2 require hospital care, which can progress to critical illness with hypoxic respiratory failure. In patients with severe COVID-19, immunomodulation with corticosteroids and IL-6 receptor antagonists has been shown to improve survival., ${ }^{1,2}$ Treatments that effectively inhibit viral replication might reduce tissue damage and allow time for the host to develop an adaptive immune response that can clear the infection. However, no treatment directed against the virus has been shown to reduce mortality (although remdesivir might shorten the duration of hospital stay). ${ }^{3}$

Humoral immunity is a key component of the immune response to SARS-CoV-2, and it matures over several weeks following infection. Anti-SARS-CoV-2 antibodies are detectable at a mean of 13 days after symptom onset, but neutralising titres do not peak until day 23, and there is wide variation in both the timing of seroconversion and peak antibody concentrations between infected individuals. ${ }^{4}$ Although patients with severe COVID-19 generally have higher final antibody concentrations than those with mild disease, their antibody responses are delayed. ${ }^{5}$ Antibodies might modulate acute viral disease either through a direct antiviral effect-by binding and neutralising free virus-or indirectly by activating antiviral pathways-such as the complement cascade, phagocytosis, and cellular cytotoxicity. Conversely, there is also a possibility that antibodies might enhance disease, either by promoting viral entry or by proinflammatory mechanisms, such as Fc $\gamma$ receptor stimulation. ${ }^{6}$

Convalescent plasma has been used for more than 100 years as passive immunotherapy for influenza pneumonia, and more recently for SARS-CoV? Although observational studies have suggested that convalescent
Lancet 2021; 397: 2049-59

Published Online May 14, 2021

https://doi.org/10.1016/ S0140-6736(21)00897-7

See Comment page 2024

*The writing committee and trial steering committee are listed at the end of this manuscript and a complete list of collaborators in the RECOVERY trial is provided in the appendix (pp 2-28)

Correspondence to: Prof Peter W Horby and Prof Martin J Landray, RECOVERY Central Coordinating Office, Oxford OX3 7LF, UK recoverytrial@ndph.ox.ac.uk See Online for appendix 
Research in context

\section{Evidence before this study}

We searched the MEDLINE, Embase, MedRxiv, and bioRxiv databases from Sept 1, 2019, to March 23, 2021, for randomised trials or meta-analyses of trials evaluating the effect of convalescent plasma in patients hospitalised with COVID-19 using the search terms ("COVID-19", "COVID", "SARS-CoV-2", "2019-nCoV", or "Coronavirus") and ("convalescent plasma", "hyperimmune plasma", "immune plasma", "passive immunization", or "plasma therapy"). 12 trials were identified. Two trials were excluded from the meta-analysis: one trial of 49 patients that did not have robust allocation concealment and one trial of 30 patients that did not report mortality. In two trials participants and clinicians were masked to treatment allocation and the remaining eight trials were open-label. There was some concern about missing outcome data in one trial, but the remaining nine studies were assessed as having a low risk of bias when using an outcome of mortality. These trials included 1495 randomly assigned patients, of whom 218 died. Most of these studies recruited patients shortly after admission to hospital, as was the case in RECOVERY.
Added value of this study

RECOVERY is the largest randomised trial to report results of the effect of convalescent plasma in patients hospitalised with COVID-19. We found that compared with usual care alone, high-titre convalescent plasma did not reduce 28-day mortality, the probability of discharge within 28 days, or the probability of progressing to the composite outcome of invasive mechanical ventilation or death in patients who were not receiving invasive mechanical ventilation at randomisation. We saw no evidence of any material benefit or hazard of convalescent plasma in any patient subgroup. Taking the results of all trials together, including RECOVERY which includes about eight-times as much information as all other trials combined, allocation to convalescent plasma was associated with a mortality rate ratio 0.98 (95\% Cl 0.91-1.06; $\mathrm{p}=0.63$ ).

Implications of all the available evidence

For patients admitted to hospital with COVID-19, convalescent plasma offers no material therapeutic benefits.
For the protocol, statistical analysis plan, and additional information see https://www. recoverytrial.net plasma might reduce mortality in severe viral respiratory infections evidence from randomised trials remains scarce and inconclusive. ${ }^{8}$ Convalescent plasma has been used widely outside of clinical trials, including by more than 100000 patients in the US Food and Drugs Administration (FDA) Expanded Access Program. ${ }^{9}$ An observational analysis of 3082 patients in this programme reported that in patients who had not received mechanical ventilation, 30-day mortality was lower in those transfused with higher-titre plasma (containing higher concentrations of anti-SARS-CoV-2 spike IgG) compared with those who received lower-titre plasma..$^{10} \mathrm{~A}$ number of randomised trials of convalescent plasma in patients hospitalised with COVID-19 have been reported, but these trials have all been small and inconclusive. ${ }^{11-20}$ Moreover, patients who are hospitalised with COVID-19 are heterogeneous and any benefit of convalescent plasma could depend on the stage of disease, perhaps being restricted to those with milder disease early in the course of their illness or those who have not mounted an effective antibody response. ${ }^{14}$ Therefore, the efficacy of convalescent plasma as a treatment for patients hospitalised with COVID-19 is uncertain. We aimed to evaluate the efficacy and safety of convalescent plasma in patients hospitalised with COVID-19.

\section{Methods}

\section{Study design and participants}

The RECOVERY trial is an investigator-initiated, individually randomised, controlled, open-label, adaptive platform trial to evaluate the effects of potential treatments in patients hospitalised with COVID-19. Details of the trial design and results for other evaluated treatments (dexamethasone, hydroxychloroquine, lopinavir-ritonavir, azithromycin, and tocilizumab) have been published previously. ${ }^{2}$ The trial is underway at 177 NHS hospital organisations in the UK (appendix pp 5-28), supported by the National Institute for Health Research Clinical Research Network. The trial was coordinated by the trial sponsor, the Nuffield Department of Population Health, University of Oxford (Oxford, UK). The trial is being done in accordance with the principles of the International Conference on Harmonisation-Good Clinical Practice guidelines and approved by the UK Medicines and Healthcare products Regulatory Agency and the Cambridge East Research Ethics Committee (20/EE/0101). The protocol, statistical analysis plan, and additional information are available online and in the appendix (pp 66-151).

Hospitalised patients of any age were eligible for the trial if they had clinically suspected or laboratoryconfirmed SARS-CoV-2 infection and no medical history that might, in the opinion of the attending clinician, put them at significant risk if they were to participate in the trial. Written informed consent was obtained from all patients or from their legal representative if they were too unwell or unable to provide consent.

\section{Randomisation and masking}

Baseline data were collected using a web-based case report form that included demographics, level of respiratory support, major comorbidities, suitability of the trial treatment for a particular patient and treatment availability at the trial site (appendix pp 35-37). Patients had a serum sample taken before random assignment for the purpose of assessing the presence of antibodies against SARS-CoV-2. 
Until Sept 18, 2020, eligible and consenting patients were randomly assigned (1:1) to receive either usual care (usual care group) or usual care plus convalescent plasma (convalescent plasma group). From Sept 18, 2020, patients were randomly assigned (1:1:1) to the usual care group, convalescent plasma group, or to receive usual care plus REGN-COV2 (a combination of two monoclonal antibodies directed against SARS-CoV-2 spike protein; appendix pp 35-37). The REGN-COV2 evaluation is ongoing and not reported here. Random assignment was unstratified and done by local clinical or research staff using a web-based interface with allocation concealment (appendix pp 33-34). For some patients, convalescent plasma was either declined, unavailable at the trial site at the time of enrolment, or considered in the opinion of the attending doctor to be definitely contraindicated (eg, known moderate or severe allergy to blood components). These patients were not included in the comparison of convalescent plasma versus usual care.

In a factorial design, patients could be simultaneously randomly assigned to other treatment groups: (1) hydroxychloroquine or dexamethasone or azithromycin or lopinavir-ritonavir or colchicine versus usual care, and (2) aspirin versus usual care (appendix pp 33-34). The trial also allowed a subsequent randomisation for patients with progressive COVID-19 (evidence of hypoxia and a hyperinflammatory state) to tocilizumab versus usual care. Participants and local study staff were not masked to the allocated treatment. Several of these treatment groups were added to or removed from the protocol over the period that convalescent plasma was evaluated (appendix pp 29-34). The trial steering committee, investigators, and all other individuals involved in the trial were masked to outcome data during the trial.

\section{Procedures}

Convalescent plasma donors were recruited and screened by the four UK blood services: NHS Blood and Transplant, the Northern Ireland Blood Transfusion Service, the Scottish National Blood Transfusion Service, and the Welsh Blood Service (appendix pp 2-4, 29). Only plasma donations with a sample to cutoff ratio of $6 \cdot 0$ or more on the EUROIMMUN IgG enzyme-linked immunosorbent assay (ELISA) targeting the spike glycoprotein (PerkinElmer, London, UK) were supplied for the RECOVERY trial (appendix p 29). EUROIMMUN IgG has been shown to correlate well with neutralisation assays, and a sample to cutoff ratio of $6 \cdot 0$ or more was previously shown to be associated with neutralising titres of 1:100 or more in convalescent plasma. ${ }^{21-24}$ The US FDA has determined that convalescent plasma with a EUROIMMUN sample to cutoff of $3 \cdot 5$ or more qualifies as high titre and can be used for the treatment of hospitalised patients under an Emergency Use Authorisation. ${ }^{9}$ Patients in the convalescent plasma group received two units (275 $\mathrm{ml}$ [200-350]) intravenously, the first as soon as possible after randomisation and the second (from a different donor) the following day and at least $12 \mathrm{~h}$ after the first.

Early safety outcomes were recorded by site staff using an online form $72 \mathrm{~h}$ after randomisation (appendix pp 38-42). An online follow-up form was completed by site staff when patients were discharged, had died, or at 28 days after randomisation, whichever occurred first (appendix pp 43-49). Information was recorded on adherence to allocated trial treatment, receipt of other COVID-19 treatments, duration of admission, receipt of respiratory or renal support, and vital status (including cause of death). In addition, routine health-care and registry data were obtained, including information on vital status at day 28 (with date and cause of death); discharge from hospital; and receipt of respiratory support or renal replacement therapy.

Baseline SARS-CoV-2 serostatus for each participant was determined using serum samples taken at the time of randomisation. Analysis was done at a central laboratory with a validated 384 well plate indirect ELISA (appendix p 29). ${ }^{25}$ Participants were categorised as seropositive or seronegative using a predefined assay threshold with a $99 \%$ or higher sensitivity and specificity in detecting individuals with SARS-CoV-2 infection at least 20 days previously. ${ }^{25}$

\section{Outcomes}

Outcomes were assessed at 28 days after randomisation, with additional analyses specified at 6 months. The primary outcome was all-cause mortality. Secondary outcomes were time to discharge from hospital and, in patients not receiving mechanical ventilation at randomisation, subsequent receipt of invasive mechanical ventilation (including extra-corporeal membrane oxygenation) or death. Prespecified, subsidiary clinical outcomes included receipt of ventilation, time to successful cessation of invasive mechanical ventilation (defined as removal of invasive mechanical ventilation within, and survival to, 28 days), and use of renal dialysis or haemofiltration.

Prespecified safety outcomes were transfusion related adverse events at $72 \mathrm{~h}$ following randomisation (worsening respiratory status, suspected transfusion reaction, fever, hypotension, haemolysis, and thrombotic events), cause-specific mortality, and major cardiac arrhythmia. Information on serious adverse reactions to convalescent plasma was collected in an expedited fashion via the existing NHS Serious Hazards of Tranfusion haemovigilence scheme.

\section{Statistical analysis}

In accordance with the statistical analysis plan, an intention-to-treat comparison was done between patients in the convalescent plasma group and those in the usual care group for whom convalescent plasma was both available and suitable as a treatment. For the primary outcome of 28-day mortality, the log-rank observed minus expected statistic and its variance were used both to test 


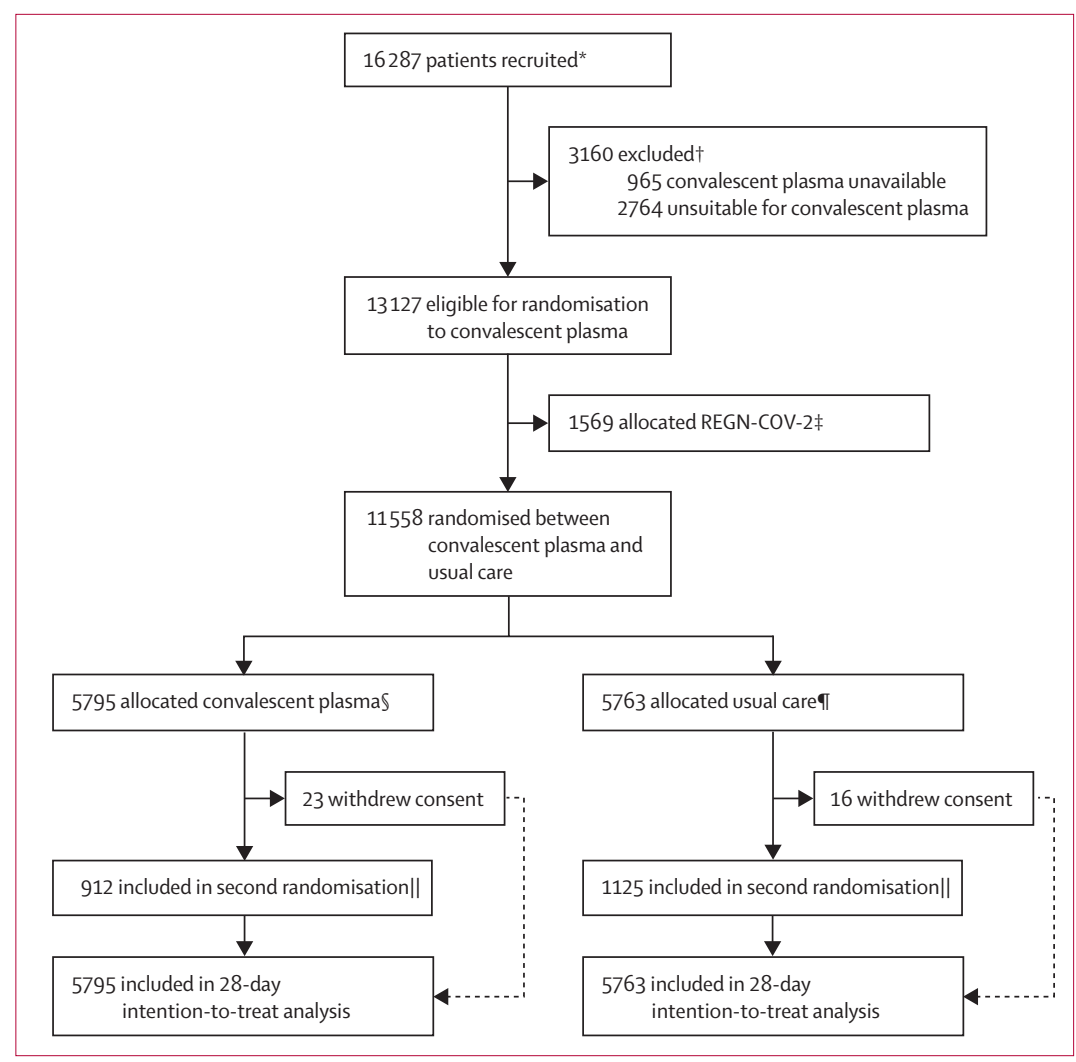

Figure 1: Trial profile

*Number recruited overall during period that patients could be recruited into convalescent plasma comparison. †Reasons for exclusion are not mutually exclusive. $¥$ Patients in the group are not included in the analyses of this study. \$5301 of 5795 patients with completed follow-up at time of analysis received convalescent plasma. $₫ 17$ of 5763 patients with completed follow-up at time of analysis received convalescent plasma. ||A second randomisation to tocilizumab versus usual care in patients with hypoxia and C-reactive protein $\geq 75 \mathrm{mg} / \mathrm{L}$ was introduced in protocol version 4.0; 426 patients in the convalescent plasma group were randomly assigned to receive tocilizumab with 486 randomly assigned to receive usual care alone; 573 patients in the usual care group were randomly assigned to receive tocilizumab with 552 randomly assigned to receive usual care alone.

the null hypothesis of equal survival curves (ie, the log-rank test) and to calculate the one-step estimate of the average mortality rate ratio. We used Kaplan-Meier survival curves to display cumulative mortality over the 28-day period. We used similar methods to analyse time to hospital discharge and successful cessation of invasive mechanical ventilation, with patients who died in hospital right-censored on day 29. Median time to discharge was derived from Kaplan-Meier estimates. For the prespecified, composite, secondary outcome of progression to invasive mechanical ventilation or death within 28 days (in those not receiving invasive mechanical ventilation at randomisation) and the subsidiary clinical outcomes of receipt of ventilation and use of haemodialysis or haemofiltration, the precise dates were not available so the risk ratio was estimated instead.

Prespecified analyses of the primary outcome were done in seven subgroups defined by characteristics at randomisation: age, sex, ethnicity, respiratory support received, days since symptom onset, use of systemic corticosteroids, and presence of anti-SARS-CoV-2 antibody. Observed effects within these subgroup categories were compared using a $\chi^{2}$ test. Subgroup analyses according to these baseline characteristics were also done for the secondary outcomes. Post-hoc exploratory analyses of the primary outcome included examination by days since symptom onset, using four subcategories rather than the two that were prespecified, and level of respiratory support by subdividing the oxygen only group into three subcategories. In late 2020, a new SARS-CoV-2 variant, named B.1.1.7, with multiple substitutions in the receptor binding domain of the spike glycoprotein emerged in southeast England and rapidly grew to become the dominant virus variant throughout the UK. ${ }^{26}$ Convalescent plasma from individuals infected before the emergence of B.1.1.7 show a modest reduction in ability to neutralise B.1.1.7 compared with earlier SARS-CoV-2 virus variants. ${ }^{27}$ The clinical significance of this reduced in-vitro neutralisation is not known. To assess if there was evidence of a difference in the effectiveness of convalescent plasma before and after the emergence of B.1.1.7, an additional post-hoc exploratory analysis was done of the primary outcome comparing effects in patients randomly assigned before Dec 1, 2020, with those randomly assigned from Dec 1,2020 , onwards. ${ }^{26}$

Additional sensitivity analyses included restricting analysis of the primary outcome to patients with a positive PCR test for SARS-COV-2 and repeating subgroup analyses of the primary and secondary outcomes by presence of anti-SARS-CoV-2 antibody after adjustment for age. Age adjustment was done because in seronegative patients those assigned to the convalescent plasma group were slightly younger than those assigned to the usual care group, whereas in seropositive patients those assigned to the convalescent plasma group were slightly older than those assigned to the usual care group. A final prespecified exploratory analysis estimated whether the effect of allocation to convalescent plasma varied depending on whether the patient was simultaneously allocated azithromycin (the only other treatment that has both already reported its results and to which substantial numbers of patients could have been assigned at the same time as they were randomly assigned to receive convalescent plasma or usual care).

Estimates of rate and risk ratios are shown with 95\% CIs. All p values are two-sided and are shown without adjustment for multiple testing. The full database is held by the trial team who pooled the data from trial sites and did the analyses at the Nuffield Department of Population Health, University of Oxford.

For the primary outcome of 28-day mortality, the results from RECOVERY were subsequently included in a meta-analysis of results from all previous randomised trials of convalescent plasma versus usual care in patients with COVID-19. For each trial, we compared the observed number of deaths among patients allocated convalescent plasma with the expected number if all 


\begin{tabular}{|c|c|c|}
\hline & $\begin{array}{l}\text { Convalescent plasma } \\
\text { group }(n=5795)\end{array}$ & $\begin{array}{l}\text { Usual care group } \\
(\mathrm{n}=5763)\end{array}$ \\
\hline Mean age, years & $63 \cdot 5(14 \cdot 7)$ & $63 \cdot 4(14 \cdot 6)$ \\
\hline \multicolumn{3}{|l|}{ Age groups } \\
\hline$<70^{*}$ & $3705(64 \%)$ & $3748(65 \%)$ \\
\hline 70-79 & $1310(23 \%)$ & $1281(22 \%)$ \\
\hline$\geq 80$ & $780(13 \%)$ & $734(13 \%)$ \\
\hline \multicolumn{3}{|l|}{ Sex } \\
\hline Men & $3643(63 \%)$ & $3787(66 \%)$ \\
\hline Woment & $2152(37 \%)$ & $1976(34 \%)$ \\
\hline \multicolumn{3}{|l|}{ Ethnicity } \\
\hline White & $4493(78 \%)$ & $4421(77 \%)$ \\
\hline $\begin{array}{l}\text { Black, Asian, and minority } \\
\text { ethnic }\end{array}$ & $833(14 \%)$ & $887(15 \%)$ \\
\hline Unknown & $469(8 \%)$ & $455(8 \%)$ \\
\hline $\begin{array}{l}\text { Median number of days since } \\
\text { symptom onset }\end{array}$ & $9(6-12)$ & $9(6-12)$ \\
\hline $\begin{array}{l}\text { Median number of days since } \\
\text { admission to hospital }\end{array}$ & $2(1-3)$ & $2(1-4)$ \\
\hline \multicolumn{3}{|l|}{ Respiratory support received } \\
\hline No oxygen received & $442(8 \%)$ & $455(8 \%)$ \\
\hline Oxygen only $\ddagger$ & $5051(87 \%)$ & $4993(87 \%)$ \\
\hline $\begin{array}{l}\text { Invasive mechanical } \\
\text { ventilation }\end{array}$ & $302(5 \%)$ & $315(5 \%)$ \\
\hline \multicolumn{3}{|l|}{ Previous diseases } \\
\hline Diabetes & $1535(26 \%)$ & $1569(27 \%)$ \\
\hline Heart disease & $1267(22 \%)$ & $1309(23 \%)$ \\
\hline Chronic lung disease & $1385(24 \%)$ & $1328(23 \%)$ \\
\hline Tuberculosis & $20(<1 \%)$ & $23(<1 \%)$ \\
\hline HIV & $17(<1 \%)$ & $19(<1 \%)$ \\
\hline Severe liver disease $\$$ & $70(1 \%)$ & $72(1 \%)$ \\
\hline Severe kidney impairment & $323(6 \%)$ & $293(5 \%)$ \\
\hline Any of the above & $3203(55 \%)$ & $3222(56 \%)$ \\
\hline
\end{tabular}

patients were at equal risk (ie, we calculated the observed minus expected statistic [o-e], and its variance $[\mathrm{v}])$. For RECOVERY, these were taken as the log-rank observed minus expected statistic and its variance but for other trials, where the exact timing of each death was not available, these were calculated from standard formulae for $2 \times 2$ contingency tables. We then combined trial results using the log of the mortality rate ratio calculated as the inverse-variance weighted average $\mathrm{S} / \mathrm{V}$ with variance $1 / \mathrm{V}$ (and hence with $95 \% \mathrm{CI} S / \mathrm{V} \pm 1 \cdot 96 / \sqrt{ } \mathrm{V}$ ), where $S$ is the sum over all trials of $(\mathrm{O}-\mathrm{E})$ and $\mathrm{V}$ is the sum over all trials of v. Analyses were done with SAS (version 9.4) and R (version 3.4).

As stated in the protocol, appropriate sample sizes could not be estimated when the trial was being planned at the start of the COVID-19 pandemic. During the trial, external data suggested that any benefits of antibody-based therapies might be higher in patients who had not raised an adequate antibody response of their own. ${ }^{14}$ Consequently, while still masked to the results of the trial, the RECOVERY steering committee determined

\begin{tabular}{|c|c|c|}
\hline & $\begin{array}{l}\text { Convalescent plasma } \\
\text { group }(n=5795)\end{array}$ & $\begin{array}{l}\text { Usual care group } \\
(n=5763)\end{array}$ \\
\hline \multicolumn{3}{|c|}{ (Continued from previous column) } \\
\hline \multicolumn{3}{|c|}{ SARS-CoV-2 PCR test result } \\
\hline Positive & $5593(97 \%)$ & $5566(97 \%)$ \\
\hline Negative & $126(2 \%)$ & $116(2 \%)$ \\
\hline Unknown & $76(1 \%)$ & $81(1 \%)$ \\
\hline \multicolumn{3}{|c|}{ Patient SARS-CoV-2 antibody test result } \\
\hline Positive & $3078(53 \%)$ & $2810(49 \%)$ \\
\hline Negative & $2016(35 \%)$ & $1660(29 \%)$ \\
\hline Missing & $701(12 \%)$ & $1293(22 \%)$ \\
\hline \multicolumn{3}{|l|}{ Corticosteroids received } \\
\hline Yes & $5370(93 \%)$ & $5311(92 \%)$ \\
\hline No & $391(7 \%)$ & $413(7 \%)$ \\
\hline Not recorded & $34(1 \%)$ & $39(1 \%)$ \\
\hline \multicolumn{3}{|c|}{ Other randomised treatments } \\
\hline Lopinavir-ritonavir & $5(<1 \%)$ & $14(<1 \%)$ \\
\hline Dexamethasone & $3(<1 \%)$ & $3(<1 \%)$ \\
\hline Hydroxychloroquine & $1(<1 \%)$ & 0 \\
\hline Azithromycin & $587(10 \%)$ & $585(10 \%)$ \\
\hline Colchicine & $792(14 \%)$ & $791(14 \%)$ \\
\hline Aspirin & $1266(22 \%)$ & $1207(21 \%)$ \\
\hline \multicolumn{3}{|c|}{$\begin{array}{l}\text { Data are mean (SD), } \mathrm{n}(\%) \text {, or median (IQR). * Includes } 26 \text { children ( }<18 \text { years). } \\
\text { †Includes } 28 \text { pregnant women. ¥Includes non-invasive ventilation. SDefined as } \\
\text { requiring ongoing specialist care. IDefined as estimated glomerular filtration rate } \\
<30 \mathrm{~mL} / \text { min per } 1.73 \mathrm{~m}^{2} \text {. }\end{array}$} \\
\hline
\end{tabular}

that the trial should enrol sufficient patients to provide at least $90 \%$ power at a two-sided $\mathrm{p}$ value of 0.01 to detect a proportional reduction in 28-day mortality of a fifth in patients with and, separately, without detectable SARS-CoV-2 antibodies at randomisation (appendix p 34).

On Jan 7, 2021, the independent data monitoring committee did a routine review of the data and recommended that the chief investigators pause recruitment to the convalescent plasma comparison in those patients receiving invasive mechanical ventilation (including extracorporeal membrane oxygenation) at the time of randomisation. At the same time, the committee recommended that recruitment to the convalescent plasma comparison continue for all other eligible patients.

On Jan 14, 2021, the data monitoring committee did another routine review of the data and notified the chief investigators that there was no convincing evidence that continued recruitment would provide conclusive proof of worthwhile mortality benefit, either overall or in any prespecified subgroup. The committee recommended that recruitment to the convalescent plasma portion of the study should cease and follow-up be completed. Enrolment of patients to the convalescent plasma comparison was closed on Jan 15, 2021, and the preliminary result for the primary outcome was made public. The trial is registered with ISRCTN, 50189673, and ClinicalTrials.gov, NCT04381936. 
Role of the funding source

The funders of the trial had no role in trial design, data collection, data analysis, data interpretation, or writing of the report.

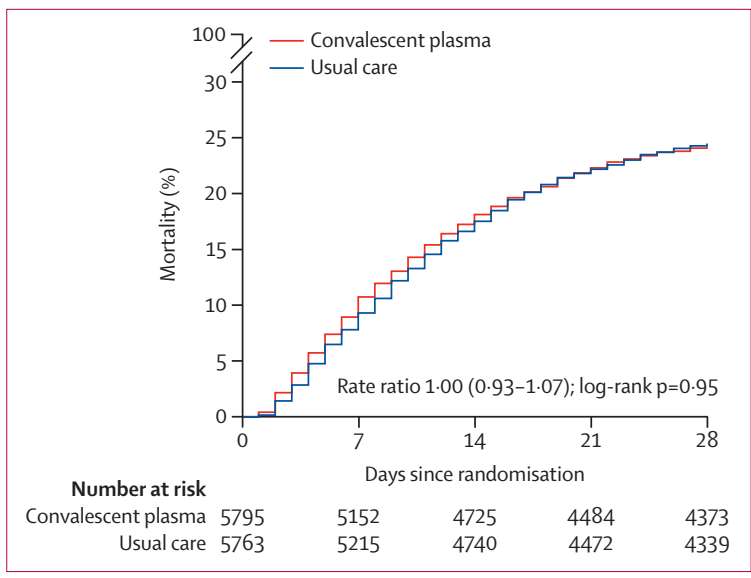

Figure 2: Effect of allocation to convalescent plasma on 28-day mortality

\section{Results}

Between May 28, 2020, and Jan 15, 2021, 13127 (81\%) of 16287 patients enrolled into the RECOVERY trial were eligible to receive convalescent plasma (figure 1). $1569(12 \%)$ were randomly assigned to the REGN-COV-2 group and are not included in the analyses reported here. Of the remaining 11558 patients, 5795 (50\%) were randomly assigned to the convalescent plasma group and $5763(50 \%)$ to the usual care group. The mean age of the patients was 63.5 (SD 14.7) years, and the median time from symptom onset to randomisation was 9 days (IQR 6-12; table 1; appendix p 51). At randomisation, 617 (5\%) of 11558 patients were receiving invasive mechanical ventilation, 10044 (87\%) were receiving oxygen only (with or without non-invasive respiratory support), and 897 (8\%) were receiving no oxygen therapy (appendix p 51). 10681 (92\%) of 11558 patients were receiving corticosteroids at time of randomisation. By chance, a slightly lower proportion of men were randomly assigned to the convalescent plasma group than the usual care group, so Cox regression analyses adjusted for sex are provided

\begin{tabular}{|c|c|c|c|c|c|}
\hline & $\begin{array}{l}\text { Convalescent } \\
\text { plasma group }\end{array}$ & $\begin{array}{l}\text { Usual care } \\
\text { group }\end{array}$ & & & $\mathrm{RR}(95 \% \mathrm{Cl})$ \\
\hline \multicolumn{6}{|l|}{ Age, years $\left(\chi_{1}^{2}=0.4 ; p=0.53\right)$} \\
\hline$<70$ & $534 / 3705(14 \%)$ & $545 / 3748(15 \%)$ & & 1 & $1.00(0.89-1.13)$ \\
\hline 70-79 & $495 / 1310(38 \%)$ & 494/1281 (39\%) & & & $0.99(0.87-1.13)$ \\
\hline$\geq 80$ & $370 / 780(47 \%)$ & $369 / 734(50 \%)$ & $\longrightarrow$ & 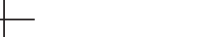 & $0.94(0.81-1.09)$ \\
\hline \multicolumn{6}{|l|}{$\operatorname{Sex}\left(\chi_{1}^{2}=1 \cdot 3 ; p=0 \cdot 25\right)$} \\
\hline Men & $953 / 3643(26 \%)$ & $972 / 3787(26 \%)$ & & & $1.03(0.95-1.13)$ \\
\hline Women & $446 / 2152(21 \%)$ & $436 / 1976(22 \%)$ & $\longrightarrow$ & - & $0.94(0.82-1.07)$ \\
\hline \multicolumn{6}{|l|}{ Ethnicity $\left(\chi_{1}^{2}=0.8 ; p=0.37\right)$} \\
\hline White & $1111 / 4493(25 \%)$ & $1129 / 4421(26 \%)$ & & & $0.97(0.90-1.06)$ \\
\hline Black, Asian or minority ethnic & $198 / 833(24 \%)$ & 199/887 (22\%) & & $\longrightarrow$ & $1.07(0.88-1.31)$ \\
\hline \multicolumn{6}{|c|}{ Days since symptom onset $\left(\chi_{1}^{2}=3 \cdot 4 ; p=0.06\right)$} \\
\hline$\leq 7$ & $606 / 2226(27 \%)$ & $660 / 2240(29 \%)$ & & & $0.92(0.82-1.03)$ \\
\hline$>7$ & $790 / 3564(22 \%)$ & $748 / 3522(21 \%)$ & & - & $1.06(0.96-1.17)$ \\
\hline \multicolumn{6}{|c|}{ Respiratory support received $\left(\chi_{1}^{2}=3.5 ; p=0.06\right)$} \\
\hline No oxygen received & $56 / 442(13 \%)$ & $69 / 455(15 \%)$ & 4 & & $0.83(0.58-1.18)$ \\
\hline Oxygen only & $1185 / 5051(23 \%)$ & $1194 / 4993(24 \%)$ & & & $0.99(0.91-1.07)$ \\
\hline Invasive mechanical ventilation & $158 / 302(52 \%)$ & $145 / 315(46 \%)$ & & $\longrightarrow$ & $1 \cdot 19(0.95-1 \cdot 50)$ \\
\hline \multicolumn{6}{|c|}{ Use of corticosteroids $\left(\chi_{1}^{2}=2 \cdot 7 ; p=0.10\right)$} \\
\hline Yes & $1314 / 5370(24 \%)$ & $1299 / 5311(24 \%)$ & & & $1.01(0.94-1.09)$ \\
\hline No & 74/391 (19\%) & $100 / 413(24 \%)$ & 4 & & $0.78(0.58-1.05)$ \\
\hline \multicolumn{6}{|c|}{ Patient SARS-CoV- 2 antibody test result $\left(\chi_{1}^{2}=1 \cdot 4 ; p=0 \cdot 23\right)$} \\
\hline Positive & $575 / 3078(19 \%)$ & $501 / 2810(18 \%)$ & & - & $1.06(0.94-1.19)$ \\
\hline Negative & $642 / 2016(32 \%)$ & $558 / 1660(34 \%)$ & & - & $0.96(0.85-1.07)$ \\
\hline Not done & $182 / 701(26 \%)$ & $349 / 1293(27 \%)$ & & & $0.98(0.82-1.17)$ \\
\hline \multirow[t]{4}{*}{ All participants } & $1399 / 5795(24 \%)$ & $1408 / 5763(24 \%)$ & & & $1.00(0.93-1.07)$ \\
\hline & & & & & $p=0.95$ \\
\hline & & & 0.6 & $\begin{array}{ccc}1 & 1 & \\
1.2 & 1.4 & 1.6\end{array}$ & \\
\hline & & & Favours convalescent plasma & Favours usual care & \\
\hline
\end{tabular}

Figure 3: Effect of allocation to convalescent plasma on 28-day mortality by prespecified characteristics at randomisation

The ethnicity, days since onset, and use of corticosteroids subgroups exclude those with missing data, but these patients are included in the overall summary. Information on use of corticosteroids was collected from June 18,2020, onwards following announcement of the results of the dexamethasone comparison from the RECOVERY trial. RR=rate ratio. 


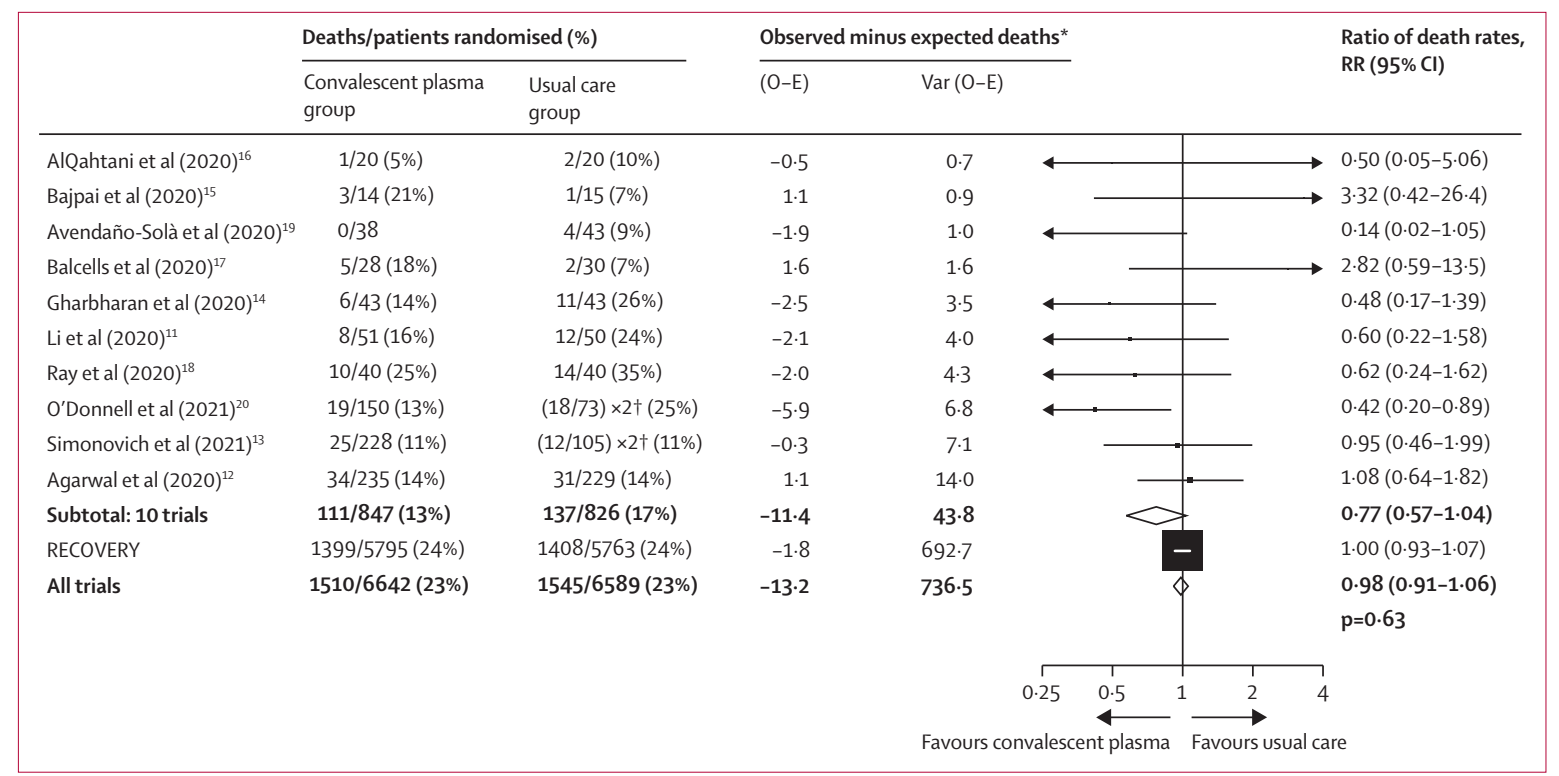

Figure 4: Meta-analysis of mortality in RECOVERY and other trials

$\mathrm{O}-\mathrm{E}=$ observed-expected. Var=variance. $\mathrm{RR}=$ rate ratio. ${ }^{*} \mathrm{Log}-$ rank $\mathrm{O}-\mathrm{E}$ for RECOVERY, $\mathrm{O}-\mathrm{E}$ from $2 \times 2$ contingency tables for the other trials. RR is calculated by taking In rate ratio to be $(\mathrm{O}-\mathrm{E}) / \mathrm{V}$ with normal variance $1 / \mathrm{V}$, where $\mathrm{V}=\operatorname{Var}(\mathrm{O}-\mathrm{E})$. Subtotals or totals of $(\mathrm{O}-\mathrm{E})$ and of $\mathrm{V}$ yield inverse-variance weighted averages of the In rate ratio values. †For balance, controls in the 2:1 studies count twice in the control totals and subtotals, but do not count twice when calculating their $\mathrm{O}$ - $\mathrm{E}$ or $\mathrm{V}$ values. Heterogeneity between RECOVERY and ten previous trials combined, $\chi_{1}{ }^{2}=2 \cdot 7(p=0 \cdot 10)$.

(appendix p 57), which are almost identical to the main results shown.

Baseline serology result were available for 9564 (83\%) of 11558 patients, of whom 3676 (38\%) were SARS-CoV-2 antibody seronegative (appendix $p$ 51). Patients were more likely to be seronegative if they were older, female, White, had shorter duration of symptoms, were receiving less intensive respiratory support, or were SARS-CoV-2 RNA negative by PCR (appendix p 52). There was an imbalance in the availability of a baseline serology sample, with more missing samples in the usual care group (table 1).

In the convalescent plasma group, 4657 (80\%) of 5795 patients received two units, 644 (11\%) received one unit, and 494 (9\%) received no units (appendix p 53). Two $(<1 \%)$ patients received both convalescent plasma units from the same donor. In the usual care group, $17(<1 \%)$ of 5763 patients received convalescent plasma. For patients in whom the time of issue of convalescent plasma by the transfusion laboratory was known, 5030 (96\%) of 5217 patients had their first unit of plasma issued within $36 \mathrm{~h}$ of randomisation. Use of corticosteroids and remdesivir following randomisation was similar between the two groups (appendix p 53). Slightly fewer patients received tocilizumab or sarilumab in the convalescent plasma group (447 [8\%] of 5795) than in the usual care group (589 [10\%] of 5763 patients; appendix p 53).

Primary and secondary outcome data were known for $99 \%$ of randomly assigned patients. There was no significant difference in 28-day mortality between the two groups: 1399 (24\%) of 5795 patients died in the convalescent plasma group and 1408 (24\%) of 5763 patients died in the usual care group (rate ratio 1.00 , 95\% CI 0.93-1.07; $\mathrm{p}=0.95$; figure 2). We observed similar results across all subgroups with no good evidence of heterogeneity of effect in either the prespecified (figure 3) or the exploratory post-hoc (appendix p 59) subgroup analyses (all p values were $>0 \cdot 05$ ). Results were similar in analyses restricted to patients with a positive SARS-CoV-2 test (rate ratio 1.00, 95\% CI 0.93-1.08; $\mathrm{p}=0 \cdot 93$ ) and there was no evidence that the rate ratio differed depending on allocation to azithromycin $(p>0 \cdot 1)$. Although 28-day mortality was higher in patients who were seronegative at randomisation, the proportional effect of allocation to convalescent plasma on 28-day mortality was similar in seropositive patients $(575$ [19\%] of 3078 patients in the convalescent plasma group vs 501 [18\%] of 2810 patients in the usual care group; rate ratio 1.06, 95\% CI 0 - 94-1.19) and seronegative patients (642 [32\%] of 2016 patients in the convalescent plasma group vs 558 [34\%] of 1660 patients in the usual care group; rate ratio 0.96 , 95\% CI 0.85-1.07; figure 3; appendix p 60). In ten other reported randomised trials, including a total of 1495 patients hospitalised with COVID-19, 218 of whom died, convalescent plasma was associated with a non-significant reduction in mortality (rate ratio 0.77 , 95\% CI 0.57-1.04; $\mathrm{p}=0 \cdot 08$; figure 4). ${ }^{11-20}$ After inclusion of the results from RECOVERY (which includes nearly eight-times as many patients and more than 11-times as many events as the other trials combined) into this 


\begin{tabular}{|c|c|c|c|c|}
\hline & $\begin{array}{l}\text { Convalescent plasma } \\
\text { group }(n=5795)\end{array}$ & $\begin{array}{l}\text { Usual care group } \\
(n=5763)\end{array}$ & $\mathrm{RR}(95 \% \mathrm{Cl})$ & $\mathrm{p}$ value \\
\hline \multicolumn{5}{|l|}{ Primary outcome } \\
\hline Mortality at 28 days & $1399(24 \%)$ & $1408(24 \%)$ & 1.00 (0.93 to 1.07$)$ & 0.95 \\
\hline \multicolumn{5}{|l|}{ Secondary outcomes } \\
\hline $\begin{array}{l}\text { Median duration of } \\
\text { hospitalisation, days }\end{array}$ & $12(6$ to $>28)$ & $11(6$ to $>28)$ &. &. \\
\hline $\begin{array}{l}\text { Discharged from hospital } \\
\text { within } 28 \text { days }\end{array}$ & $3832(66 \%)$ & $3822(66 \%)$ & 0.99 (0.94 to 1.03$)$ & 0.57 \\
\hline $\begin{array}{l}\text { Invasive mechanical } \\
\text { ventilation or death* }\end{array}$ & $1568 / 5493(29 \%)$ & $1568 / 5448(29 \%)$ & 0.99 (0.93 to 1.05$)$ & 0.79 \\
\hline $\begin{array}{l}\text { Invasive mechanical } \\
\text { ventilation }\end{array}$ & $678 / 5493(12 \%)$ & $690 / 5448(13 \%)$ & 0.97 (0.88 to 1.08$)$ & 0.61 \\
\hline Death & $1241 / 5493(23 \%)$ & $1263 / 5448(23 \%)$ & 0.97 (0.91 to 1.04$)$ & 0.46 \\
\hline \multicolumn{5}{|l|}{ Subsidiary outcomes } \\
\hline Use of ventilation $†$ & $885 / 3564(25 \%)$ & $876 / 3441(25 \%)$ & 0.98 (0.90 to 1.06$)$ & 0.55 \\
\hline Non-invasive ventilation & $856 / 3564(24 \%)$ & $845 / 3441(25 \%)$ & 0.98 (0.90 to 1.06$)$ & 0.60 \\
\hline $\begin{array}{l}\text { Invasive mechanical } \\
\text { ventilation }\end{array}$ & $229 / 3564(6 \%)$ & $238 / 3441(7 \%)$ & 0.93 (0.78 to 1.11$)$ & 0.41 \\
\hline $\begin{array}{l}\text { Successful cessation of } \\
\text { invasive mechanical } \\
\text { ventilation } \neq\end{array}$ & $85 / 302(28 \%)$ & 108/315 (34\%) & $0.79(0.59$ to 1.05$)$ & 0.11 \\
\hline Renal replacement therapy & $250 / 5707(4 \%)$ & $241 / 5697(4 \%)$ & 1.04 (0.87 to 1.23$)$ & 0.69 \\
\hline \multicolumn{5}{|c|}{$\begin{array}{l}\text { Data are } n(\%) \text {, median (IQR) or } \mathrm{n} / \mathrm{N}(\%) \text {. RR=rate ratio for the outcomes of } 28 \text {-day mortality, hospital discharge, and } \\
\text { successful cessation of invasive mechanical ventilation, and risk ratio is calculated for all other outcomes. }{ }^{*} \text { Analyses } \\
\text { exclude those on invasive mechanical ventilation at randomisation. † Analyses exclude those on invasive or } \\
\text { non-invasive ventilation at randomisation. \#Analyses exclude those not receiving invasive mechanical ventilation at } \\
\text { randomisation. SAnalyses exclude those on renal replacement therapy at randomisation. }\end{array}$} \\
\hline
\end{tabular}

meta-analysis, the mortality rate ratio was 0.98 (95\% CI $0 \cdot 91-1 \cdot 06 ; \mathrm{p}=0 \cdot 63$; figure 4).

The median time to discharge was 12 days in the convalescent plasma group and 11 days in the usual care group (IQR 6 to $>28$ in both groups); patients in the convalescent plasma group had a similar probability of being discharge alive within 28 days compared with the usual care group (3832 [66\%] of 5795 patients in the convalescent plasma group vs 3822 [66\%] of 5763 patients in the usual care group; rate ratio $0.99,95 \% \mathrm{CI}$ 0.94 to $1.03 ; \mathrm{p}=0.57$; table 2 ). Of the patients who were not receiving invasive mechanical ventilation at baseline, the number of patients progressing to the prespecified composite secondary outcome of invasive mechanical ventilation or death was similar in the two groups (1568 [29\%] of 5493 patients in the convalescent plasma group vs 1568 [29\%] of 5448 patients in the usual care group; rate ratio $0.99,95 \%$ CI 0.93 to 1.05 ; $=0.79$; table 2 ). For both of these secondary outcomes, there was some evidence of heterogeneity by patient SARS-CoV-2 antibody test result, with slightly more favourable outcomes with convalescent plasma in patients who were seronegative at baseline compared with those who were seropositive (appendix pp 61-62). Because of slight age-imbalances between treatment groups for both seropositive and seronegative patients, an exploratory analysis was done that included adjustment for age, which marginally reduced the apparent heterogeneity (heterogeneity $\mathrm{p}=0.02$ for both secondary outcomes after age adjustment). Results were consistent across all other prespecified subgroups of patients.

There were no significant differences in the prespecified subsidiary clinical outcomes of use of ventilation, successful cessation of invasive mechanical ventilation, or progression to use of renal replacement therapy (table 2), or in cause-specific mortality (appendix p 54).

Within the first $72 \mathrm{~h}$ after randomisation, severe allergic reactions were reported in $16(<1 \%)$ of 5795 patients in the convalescent plasma group and two $(<1 \%)$ of 5763 patients in the usual care group. The frequency of sudden worsening in respiratory status, temperature higher than $39^{\circ} \mathrm{C}$ or a $2^{\circ} \mathrm{C}$ or higher increase in temperature above baseline, sudden hypotension, clinical haemolysis, and thrombotic events were broadly similar in the two groups (appendix p 55). We also observed no significant differences in the frequency of major cardiac arrhythmia (appendix p 56). 13 patients receiving convalescent plasma had reports submitted to the Serious Hazards of Transfusion haemovigilence scheme: nine patients with pulmonary reactions (none considered to be transfusion-related acute lung injury, including three deaths possibly related to transfusion), and four patients with serious febrile, allergic, or hypotensive reactions (all of whom recovered).

\section{Discussion}

The results of this large, randomised trial show that convalescent plasma did not improve survival or other clinical outcomes in patients hospitalised with COVID-19. The results were consistent across subgroups of age, sex, ethnicity, duration of symptoms before randomisation, level of respiratory support received at randomisation, and use of corticosteroids. The results are consistent with the evidence from previously reported randomised trials of convalescent plasma for patients hospitalised with COVID-19, ${ }^{11-20}$ with no evidence of a survival benefit when these results are combined (figure 4).

It has been suggested that the benefits of convalescent plasma depend on the transfused neutralising titre, and that using plasma with lower titres could explain negative results from previous randomised trials. In RECOVERY, all convalescent plasma was supplied via the UK National Blood Services using standardised laboratory processing. Convalescent donors were chosen based on high anti-spike IgG concentrations, using an ELISA that has been shown to correlate well with neutralising antibody. ${ }^{22-24}$ We used a EUROIMMUN sample to cutoff ratio of $6 \cdot 0$ for plasma to qualify for use in this trial, which is substantially more than the $3 \cdot 5$ cutoff that the US FDA recognises as high titre. ${ }^{9}$ Nearly all participants received plasma from two different donors to increase the chance that at least one contained higher concentrations of neutralising antibodies. 
The presence of anti-SARS-CoV-2 antibodies in recipients at the time of transfusion with convalescent plasma has also been cited as a possible reason for the absence of an observed effect. ${ }^{14}$ In this trial we found that $38 \%$ of patients were seronegative at randomisation, and, although they had a markedly higher 28-day mortality risk than patients who were seropositive at randomisation, we did not observe a significant survival benefit from convalescent plasma in these patients. Our results do not exclude the possibility of small improvements in the probability of successful discharge from hospital by day 28 or of progressing to invasive mechanical ventilation or death in seronegative patients who received convalescent plasma. However, the results of these secondary outcomes in one subgroup should be interpreted with caution given the multiple testing; additionally, when an age-adjusted analysis was done the apparent heterogeneity was slightly reduced.

It has also been suggested that antibody-based therapies could be most effective in the early stages of COVID-19, when viral replication dominates. ${ }^{10,28}$ We did not identify a benefit of convalescent plasma when patients were stratified by time since onset of illness in the main analysis or in an exploratory analysis, which subdivided participants on the basis of illness onset. Of note, we did not identify a mortality benefit in the subgroup of patients allocated to convalescent plasma 4 days or less from illness onset, which by itself comprised more patients than the total number of patients enrolled in all other convalescent plasma trials combined. However, RECOVERY only included patients admitted to hospital; therefore, the trial does not address whether convalescent plasma has any benefit if given early after SARS-CoV-2 infection and before the onset of significant disease. That question has not yet been robustly tested in sufficiently large randomised controlled trials. ${ }^{28}$

Following random assignment to receive convalescent plasma, patients with hypoxia and a raised C-reactive protein $(\geq 75 \mathrm{mg} / \mathrm{L})$ were eligible for a second random assignment to receive usual care or usual care plus tocilizumab. Although a slightly lower proportion of patients allocated convalescent plasma (8\%) subsequently received tocilizumab than patients allocated usual care (10\%; appendix p 53), and although tocilizumab itself reduces 28-day mortality by around 15\%, ${ }^{1}$ this difference is far too small to have had any material effect on our estimate of the effect of convalescent plasma on mortality (estimated $0 \cdot 1 \%$ difference in 28 day mortality).

SARS-CoV-2 is an RNA virus with antigenic variability. The efficacy of convalescent plasma is likely to depend on the match between the strain-specific transfused antiSARS-CoV-2 antibodies in donor plasma and the infecting virus variant in the recipient. In December, 2020, a new SARS-CoV-2 variant (B.1.1.7) was detected in the southeast and east of England, with an earliest date of detection in September, 2020. B.1.1.7 spread rapidly to become the dominant SARS-CoV-2 variant, in most regions of the UK, by January, 2021. ${ }^{29}$ Although B.1.1.7 has changes in the spike glycoprotein that could theoretically modify antigenicity, only modest reductions in neutralisation by convalescent plasma have been reported. ${ }^{27}$ Consistent with this, we did not identify any evidence of a differential effect of convalescent plasma before and after the emergence of B.1.1.7 in the UK (appendix p 59).

During an epidemic caused by a novel virus, convalescent plasma is an appealing treatment because it might be available within weeks of the outbreak, long before other targeted therapies are available. Consequently, convalescent plasma has been widely used for COVID-19 outside of clinical trials but, until now, there has been insufficient evidence from randomised trials to reliably assess its safety and efficacy. ${ }^{10}$ In RECOVERY, the largest clinical trial of convalescent plasma for any infectious indication, we did not find evidence that high-titre convalescent plasma improved survival or other prespecified clinical outcomes in patients hospitalised with COVID-19. Whether convalescent plasma would benefit other patient groups is unknown and would need to be evaluated in other, adequately powered, randomised clinical trials.

\section{Contributors}

This manuscript was initially drafted by the PWH and MJL, further developed by the Writing Committee, and approved by all members of the trial steering committee. PWH and MJL vouch for the data and analyses, and for the fidelity of this report to the trial protocol and data analysis plan. PWH, LE, LP, MM, JKB, LCC, SNF, TJ, KJ, WSL, AM, KR, EJ, DJR, RH, and MJL designed the trial and trial protocol. MM, AR, GP-A, NJB, TG, DZ, ST, NNA, AU, JW, GK, TB, SS, RH, the Data Linkage team at the RECOVERY Coordinating Centre, and the health records and local clinical centre staff listed in the appendix collected the data. ES, NS, and JRE did the statistical analysis. LE, DJR, and the blood and transfusion service staff listed in the appendix coordinated the collection and supply of convalescent plasma. All authors contributed to data interpretation and critical review and revision of the manuscript. PWH and MJL had access to the trial data and had final responsibility for the decision to submit for publication.

Writing Committee (on behalf of the RECOVERY Collaborative Group) Peter W Horby, Lise Estcourt, Leon Peto, Jonathan R Emberson, Natalie Staplin, Enti Spata, Guilherme Pessoa-Amorim, Mark Cambell, Alistair Roddick, Nigel J Brunskill, Tina George, Daniel Zehnder, Simon Tiberi, Ni Ni Aung, Alison Uriel, John Widdrington, George Koshy, Thomas Brown, Stephen Scott, J Kenneth Baillie, Maya H Buch, Lucy C Chappell, Jeremy N Day, Saul N Faust, Thomas Jaki, Katie Jeffery, Edmund Juszczak, Wei Shen Lim, Marion Mafham, Alan Montgomery, Andrew D Mumford, Kathryn Rowan, Guy Thwaites, David J Roberts, Richard Haynes, Martin J Landray. PWH, LE, and LP contributed equally, and DJR, RH, and MJL contributed equally.

\section{Data Monitoring Committee}

Peter Sandercock, Janet Darbyshire, David DeMets, Robert Fowler, David Lalloo, Ian Roberts (until December 2020), Mohammed Munavvar (from January 2021), Janet Wittes.

\section{Declaration of interests}

We declare no competing interests.

Data sharing

The protocol, consent form, statistical analysis plan, definition and derivation of clinical characteristics and outcomes, training materials, regulatory documents, and other relevant trial materials are available online. As described in the protocol, the trial steering committee will facilitate the use of the trial data and approval will not be unreasonably withheld. Deidentified participant data will be made available to bona fide researchers registered with an appropriate institution within 
For data policy and procedures see https://www.ndph.ox.ac.uk/ data-access For the access from see https:// www.ndph.ox.ac.uk/files/about/ data access enquiry form_13_6_2019.docx
3 months of publication. However, the steering committee will need to be satisfied that any proposed publication is of high quality, honours the commitments made to the trial patients in the consent documentation and ethical approvals, and is compliant with relevant legal and regulatory requirements (eg, relating to data protection and privacy). The steering committee will have the right to review and comment on any draft manuscripts before publication. Data will be made available in line with the policy and procedures available online. Those wishing to request access should complete the form available online and emailed to data. access@ndph.ox.ac.uk.

\section{Acknowledgments}

We would like to thank the thousands of patients who participated in this trial. We would also like to thank the many doctors, nurses, pharmacists, other allied health professionals, and research administrators at 177 NHS hospital organisations across the whole of the UK, supported by staff at the National Institute of Health Research (NIHR) Clinical Research Network, NHS DigiTrials, NHS Blood and Transplant, the Scottish National Blood Transfusion Service, the Welsh Blood Service, the Northern Ireland Blood Transfusion Service, Public Health England, Department of Health \& Social Care, the Intensive Care National Audit \& Research Centre, Public Health Scotland, National Records Service of Scotland, the Secure Anonymised Information Linkage at University of Swansea, Swansea, UK, and the NHS in England, Scotland, Wales and Northern Ireland. The RECOVERY trial is supported by a grant to the University of Oxford from UK Research and Innovation (UKRI) and NIHR (MC_PC_19056), by the Department of Health and Social Care (DHSC), UKRI, and NIHR COVID-19 Rapid Response Grant (COV19-RECPLA), and by core funding provided by NIHR Oxford Biomedical Research Centre, the Wellcome Trust, the Bill \& Melinda Gates Foundation, the Department for International Development, Health Data Research UK, the Medical Research Council Population Health Research Unit, the NIHR Health Protection Unit in Emerging and Zoonotic Infections, NHS Blood and Transplant Research and Development Funding, EU's Horizon 2020 research and innovation programme (SUPPORT-E - 101015756), and NIHR Clinical Trials Unit Support Funding. TJ is supported by a grant from UK Medical Research Council (MC_UU_0002/14) and an NIHR Senior Research Fellowship (NIHR-SRF-2015-08-001). WSL is supported by core funding provided by NIHR Nottingham Biomedical Research Centre. AbbVie contributed some supplies of lopinavir-ritonavir for use in this trial. Tocilizumab was provided free of charge for this trial by Roche. REGN-COV2 was provided free of charge for this trial by Regeneron. The collection of plasma was funded by the DHSC through core funding and funding under COVID-19 and EU SoHo Grant. The views expressed in this publication are those of the authors and not necessarily those of the NHS, the NIHR, NHS Blood and Transplant, the DHSC, or the EU.

\section{References}

1 RECOVERY Collaborative Group. Tocilizumab in patients admitted to hospital with COVID-19 (RECOVERY): a randomised controlled, open-label, platform trial. Lancet 2021; 397: 1637-45.

2 RECOVERY Collaborative Group, Horby P, Lim WS, et al Dexamethasone in hospitalized patients with Covid-19. N Engl J Med 2021; 384: 693-704.

3 Beigel JH, Tomashek KM, Dodd LE, et al. Remdesivir for the treatment of Covid-19-final report. N Engl J Med 2020; 383: 1813-26.

4 Seow J, Graham C, Merrick B, et al. Longitudinal observation and decline of neutralizing antibody responses in the three months following SARS-CoV-2 infection in humans. Nat Microbiol 2020; 5: 1598-607.

5 Li K, Huang B, Wu M, et al. Dynamic changes in anti-SARS-CoV-2 antibodies during SARS-CoV-2 infection and recovery from COVID-19. Nat Commun 2020; 11: 6044

6 Lee WS, Wheatley AK, Kent SJ, DeKosky BJ. Antibody-dependent enhancement and SARS-CoV-2 vaccines and therapies. Nat Microbiol 2020; 5: 1185-91.

7 Mair-Jenkins J, Saavedra-Campos M, Baillie JK, et al. The effectiveness of convalescent plasma and hyperimmune immunoglobulin for the treatment of severe acute respiratory infections of viral etiology: a systematic review and exploratory meta-analysis. J Infect Dis 2015; 11: 80-90.
8 Devasenapathy N, Ye Z, Loeb M, et al. Efficacy and safety of convalescent plasma for severe COVID-19 based on evidence in other severe respiratory viral infections: a systematic review and meta-analysis. CMAJ 2020; 192: E745-55.

9 US Department of Health and Human Services Food and Drug Administration. Letter of Authorization, Reissuance of Convalescent Plasma EUA. 2021. https://www.fda.gov/media/141477/download (accessed April 27, 2021)

10 Joyner MJ, Carter RE, Senefeld JW, et al. Convalescent plasma antibody levels and the risk of death from Covid-19. N Engl J Med 2021; 384: 1015-27.

11 Li L, Zhang W, Hu Y, et al. Effect of convalescent plasma therapy on time to clinical improvement in patients with severe and lifethreatening COVID-19: a randomized clinical trial. JAMA 2020; 324: 460-70.

12 Agarwal A, Mukherjee A, Kumar G, et al. Convalescent plasma in the management of moderate covid-19 in adults in India: open labe phase II multicentre randomised controlled trial (PLACID Trial). BMJ 2020; 371: m3939.

13 Simonovich VA, Burgos Pratx LD, Scibona P, et al. A randomized trial of convalescent plasma in Covid-19 severe pneumonia. N Engl J Med 2021; 384: 619-29.

14 Gharbharan A, Jordans CCE, GeurtsvanKessel C, et al. Convalescen plasma for COVID-19. A randomized clinical trial. medRxiv 2020 published online July 3. https://doi.org/10.1101/2020.07.01.20139857 (preprint)

15 Bajpai M, Kumar S, Maheshwari A, et al. Efficacy of convalescentplasma therapy compared to fresh frozen plasma in severely ill COVID-19 patients: a pilot randomized controlled trial. medRxiv 2020; published onoine Oct 27. https://doi.org/10.1101/ 2020.10.25.20219337 (preprint).

16 AlQahtani M, Abdulrahman A, Almadani A, et al. Randomized controlled trial of convalescent plasma therapy against standard therapy in patients with severe COVID-19 disease. medRxiv 2020; published online Nov 4. https://doi.org/10.1101/2020.11.02.20224303 (preprint)

17 Balcells ME, Rojas L, Le Corre N, et al. Early anti-SARS-CoV-2 convalescent plasma in patients admitted for COVID-19: a randomized phase II clinical trial. PLoS Med 2020; 18: e1003415.

18 Ray Y, Paul SR, Bandopadhyay P, et al. Clinical and immunological benefits of convalescent plasma therapy in severe COVID-19: insights from a single center open label randomised control trial. medRxiv 2020; published online Nov 29. https://doi.org/10.1101/ 2020.11.25.20237883 (preprint)

19 Avendaño-Solà C, Ramos-Martínez A, Muñez-Rubio E, et al. Convalescent Plasma for COVID-19: a multicenter, randomized clinical trial. medRxiv 2020; published online Sept 29. https://doi. org/10.1101/2020.08.26.20182444 (preprint).

20 O'Donnell MR, Grinsztejn B, Cummings MI, et al. A randomized, double-blind, controlled trial of convalescent plasma in adults with severe COVID-19. medRxiv 2021; published online March 13. https://doi.org/10.1101/2021.03.12.21253373 (preprint).

21 Harvala H, Mehew J, Robb ML, et al. Convalescent plasma treatment for SARS-CoV-2 infection: analysis of the first 436 donors in England, 22 April to 12 May 2020. Euro Surveill 2020; 25: 2001260.

22 Patel EU, Bloch EM, Clarke W, et al. Comparative performance of five commercially available serologic assays to detect antibodies to SARS-CoV-2 and identify individuals with high neutralizing titers. J Clin Microbiol 2020; 59: e02257-20.

23 Walker GJ, Naing Z, Ospina Stella A, et al. SARS coronavirus-2 microneutralisation and commercial serological assays correlated closely for some but not all enzyme immunoassays. Viruses 2021; 13: 247.

24 Valdivia A, Torres I, Latorre V, et al. Inference of SARS-CoV-2 spike-binding neutralizing antibody titers in sera from hospitalized COVID-19 patients by using commercial enzyme and chemiluminescent immunoassays. Eur J Clin Microbiol Infect Dis 2021; 40: 485-94.

25 National Sars-CoV-2 Serology Assay Evaluation Group. Performance characteristics of five immunoassays for SARS-CoV-2: a head-tohead benchmark comparison. Lancet Infect Dis 2020; 20: 1390-400.

26 Davies NG, Abbott S, Barnard RC, et al. Estimated transmissibility and impact of SARS-CoV-2 lineage B.1.1.7 in England. Science 2021; 372: eabg 3055 
Articles

27 Supasa P, Zhou D, Dejnirattisai W, et al. Reduced neutralization of SARS-CoV-2 B.1.1.7 variant by convalescent and vaccine sera. Cell 2021; 184: 2201-21.

28 Libster R, Perez Marc G, Wappner D, et al. Early high-titer plasma therapy to prevent severe Covid-19 in older adults. $N$ Engl J Med 2021; 384: 610-18.
29 Volz E, Mishra S, Chand M, et al. Transmission of SARS-CoV-2 lineage B.1.1.7 in England: insights from linking epidemiological and genetic data. medRxiv 2021; published online Jan 4. https://doi. org/10.1101/2020.12.30.20249034 (preprint). 